\title{
Los desafíos de enseñar historia en la formación inicial en Estudios Sociales. Un estudio de caso en el contexto costarricense
}

\author{
The challenges of teaching history in initial training in Social Studies. \\ A case study in the Costa Rican context \\ Jéssica RamíreZ Achoy ${ }^{a} \&$ Joan Pagès Blanch ${ }^{\mathrm{b}}$ \\ aUniversidad Nacional, San José, Costa Rica. \ jessica.ramirez.achoy@una.cr \\ [orcid.org/0000-0002-1795-3006] \\ ${ }^{b}$ Universidad Autónoma de Barcelona, Barcelona, España. \\ [orcid.org/0000-0001-8650-3976]
}

\section{RESUMEN}

En el artículo se estudia qué pasa en las clases de historia de la carrera de Estudios Sociales, para ello se caracterizaron las prácticas docentes de cuatro profesoras de historia y se contrapusieron con las experiencias estudiantiles del profesorado en formación. Se utilizó la metodología cualitativa con el enfoque del estudio de casos, para profundizar en el contexto y las particularidades de la Escuela de Historia, de la Universidad Nacional (UNA), en Costa Rica. El análisis de la información se realizó a través de la codificación temática y teórica, donde se fragmentaron los datos para obtener nuevos códigos que le dieran respuesta al problema de la investigación. Las evidencias mostraron las contradicciones de las dinámicas de aula, pues las finalidades de la enseñanza no van acordes a las metodologías y formas de evaluar los aprendizajes, lo cual incide en el desarrollo del pensamiento histórico del estudiantado.

PALABRAS CLAVE: didáctica de la historia, pensamiento histórico, formación inicial docente, prácticas docentes universitarias.

\section{ABSTRACT}

The article analyzes what happens in the classes of the Social Studies career when teaching history, for this, the teaching practices of four history teachers were characterized and they were contrasted with the student's experiences. The qualitative methodology was used with the focus of the case study, to deepen the context and the particularities of the Department of History, of the Universidad Nacional (UNA), in Costa Rica. The information was coding through Thematic and Theoretical Coding, where the data was fragmented to obtain 
new codes that would give an answer to the research problem. The evidence showed the contradictions of the classroom dynamics, since the purposes of teaching are not consistent with the methodologies and ways of evaluating learning, which affects the development of the Historical Thinking of the students.

KEY WORDS: didactisc of history, historical thinking, initial teacher training, teaching practices at university.

\section{INTRODUCCIÓN}

En el artículo se analiza el siguiente problema de investigación: ¿Cómo se enseña historia al futuro profesorado de Estudios Sociales de Costa Rica y si estas metodologías permiten el desarrollo del pensamiento histórico? El objetivo es caracterizar y analizar las prácticas docentes universitarias y el pensamiento histórico, para lo cual se trabajó con docentes universitarias y estudiantes de la carrera bachillerato en la Enseñanza de los Estudios Sociales y Educación Cívica, administrada por la Escuela de Historia de la Universidad Nacional, en Costa Rica. Esta investigación es parte de la tesis El desarrollo del pensamiento histórico en la formación inicial docente. Un estudio de caso en la Universidad Nacional, Costa Rica, del Doctorado en Didáctica de la Universidad Tecnológica de Pereira y fue dirigida por el Dr. Joan Pagès Blanch (Q.E.P.D.).

El pasado es un conocimiento socialmente útil en la medida que nos explique el presente y su contexto en las democracias, por tanto, se indagó qué tan crítica es la historia que se enseña en las Universidades y sus aportes para el aprendizaje del pensamiento histórico (PH). Sobre lo anterior, Armento (2000) ha señalado la necesidad de conocer cómo se forma a las y los docentes de Estudios Sociales a través de datos que nos expliquen desde el aula universitaria qué tipo de historia le enseñamos al profesorado en formación.

El problema que estudiamos en el artículo se centra en la importancia de conocer qué pasa desde el aula universitaria cuando enseñamos una disciplina, y si estos procesos promueven la interpretación del pasado a partir de la evidencia. El tema se ha empezado a estudiar y los datos indican que el futuro profesorado no aprende a pensar históricamente, pues su formación se centra en la disciplina y no en cómo pensarla ni enseñarla (Ramírez \& Pagès, 2019; Chávez \& Pagès, 2019).

En la actualidad se requieren estudios que nos permitan comprender cómo se enseña el pasado en todos los niveles educativos. La utilización de modelos conceptuales basados en el pensamiento histórico y la conciencia histórica ha dado un giro en la forma de concebir los propósitos de enseñar el pasado, y ha ampliado los objetos de estudio de la didáctica de la historia. En este sentido, Duquette (2014) afirma: “A fundamental understanding of historical thinking seems essential for student teachers who need this information to comprehend the many demands of the curriculum and implement a more active form of learning in their own future classroom" (p. 154).

Por tanto, pensar históricamente lo entendemos como "la acción de significar el pasado a través del uso deliberado de estrategias creadas por los historiadores como son el tiempo 
histórico, la causalidad, la objetividad, la intertextualidad y se representa en una forma escriturística particular" (Plá, 2005, p. 16). Sobre esta idea, importan las construcciones que cada sujeto realiza del pasado, más allá de los discursos dados sobre ese pasado. La historia ha de ser comprendida desde las narrativas y los contextos socioculturales que le permiten al sujeto interpretar el mundo o los mundos en los que vivimos.

Por lo anterior, se utilizaron las dimensiones de pensamiento histórico elaboradas por Seixas y Morton (2013) para el análisis y la interpretación de la historia. Los seis conceptos propuestos por los autores son: a) ¿Cómo decidimos qué es importante aprender del pasado? (significado histórico), b) ¿Cómo sabemos lo que sabemos del pasado? (evidencia), c) ¿Cómo podemos dar sentido a los complejos cursos de la historia? (cambio y continuidad), d) ¿Por qué suceden los procesos históricos y cuáles son sus impactos? (causas y consecuencias), e) ¿Cómo podemos entender mejor a la gente del pasado? (perspectiva histórica) y f) ¿Cómo puede la Historia ayudarnos a vivir en el presente? (dimensión ética).

Por otra parte, la formación inicial se entendió desde el modelo de Armento (2000), quien propuso estudiar las dinámicas del aula universitaria a través de la interrelación entre el currículo, el profesorado y el estudiantado. De esta forma, Armento (1996) parte de tres dimensiones para la formación inicial docente: a) el desarrollo profesional, entendido como el tránsito entre el profesor o profesora novata y la experta; b) los factores contextuales, sociales, políticos, culturales y económicos del desarrollo profesional y c) las interacciones entre docente-estudiantes-currículo.

En la formación inicial docente se conjugan tres actores: el profesor o profesora en formación, los educadores y educadoras de ese profesorado y los programas de educación o planes de estudio. Esa tríada se resume en la interrelación entre estudiantes-docentescurrículo de la Universidad, donde el contexto es fundamental para ubicar los aprendizajes que obtiene el profesorado en formación.

De esta forma, el contenido histórico es pieza fundamental de la didáctica de la historia y para su análisis se recurrió a los trabajos de Shulman (2000) y su concepto del conocimiento pedagógico del contenido, el cual es descrito como el saber disciplinar del docente aplicado a su contexto de aula. El contenido disciplinar se diferencia del contenido escolar por sus propósitos y finalidades. Mattozzi (1999) y Pagès (2018) establecen que las diferencias entre ambos inciden en la formación inicial docente (FID), pues ésta se basa en el saber disciplinar, pero no en su enseñanza.

Siguiendo esta línea, Libâneo (2012) afirma que el contenido es en sí mismo una categoría compleja:

el contenido es el conjunto de conocimientos teóricos de una disciplina considerados importantes para la formación general de los estudiantes conforme a sus edades. Tales contenidos, como regla general, son determinados por los órganos del gobierno. Pero, esos contenidos son formulados para que los estudiantes los aprendan, o sea, los conceptos implicados en los contenidos son enseñados a los estudiantes para que se conviertan en medios de su propia actividad. Donde se 
requiere, por parte de las escuelas y profesores, la “conversión” de la ciencia en materia de enseñanza (pp. 27-28).

Esta conversión tiene implicaciones sobre la enseñanza de una disciplina, pues el contenido no puede limitarse a los métodos o teorías que particularizan sus distintos campos de estudio. Al formar maestros y maestras, las universidades deben ser conscientes de que el propósito es la enseñanza y, por tanto, las teorías y métodos de cada disciplina deben de estar en función de cómo aplicarlo al aula. De lo contrario, tendremos docentes que manejan contenidos, pero no sabrán cómo enseñarlos.

La formación de las profesoras y profesores es un área que implica la reflexión del papel de la Universidad y de las disciplinas de las ciencias sociales en la promoción de docentes que piensen críticamente y, con esto, que piensen en los desafíos sociales a los que nos enfrentamos dentro de las democracias latinoamericanas. Un o una docente de Estudios Sociales no podrá enseñar ni ejercer una ciudadanía crítica sino la comprende y, en esta tarea, el pensamiento histórico, que es pensamiento creativo y crítico, constituye referencias para pensar la sociedad más allá de los datos factuales y de un pasado estático sin relación con nuestro presente y nuestro futuro. Construir ciudadanía también implica reconocerse como sujeto político dentro de un espacio social, en el cual la identidad colectiva y la memoria tienen un papel trascendental en esta tarea.

\section{METODOLOGÍA}

Metodológicamente se trabajó desde la investigación cualitativa con el método de estudio de casos, con la finalidad de profundizar en las experiencias docentes y estudiantiles en las clases de historia. Los marcos teóricos que fundamentan la investigación cualitativa son diversos, sin embargo, también "muestran que las personas, las instituciones y las interacciones están implicadas en la producción de las realidades en las que viven o se producen, y que estas tareas de producción se basan en procesos de creación de significado" (Flick, 2007, p. 32). En este sentido, se estudiaron los significados que las historiadoras de la Escuela de Historia les dan a sus prácticas de enseñanza en la carrera de Estudios Sociales y cómo el estudiantado experimenta los procesos de aprendizaje del pasado.

Los sujetos del estudio fueron cuatro historiadoras que dan clases en la carrera de Estudios Sociales y 8 estudiantes de distintos niveles. Para obtener la información, se aplicaron las técnicas de recolección de datos, propias del estudio de caso. En total se realizaron ocho observaciones de aula universitaria, tres entrevistas abiertas a las profesoras y un grupo de discusión con estudiantes. También se analizaron documentos curriculares, tales como los programas de curso que elaboró el profesorado, las evaluaciones aplicadas durante el I semestre, los trabajos que presentó el estudiantado en los cursos y del plan de estudios de la carrera. Todo el proceso de recolección de datos se realizó entre los meses de febrero y julio del año 2019.

Los parámetros utilizados en la guía de observación fueron: ¿Qué sucede cuando se enseña y se aprende y por qué sucede lo que sucede? ¿Cómo se introducen los contenidos, 
cómo se desarrollan y cómo se concluye la clase? ¿Cuál es la metodología en las actividades de enseñanza? ¿Cuál es el protagonismo docente? ¿Cuánto tiempo dura la atención del alumnado a la exposición del docente? ¿Cuándo y cómo se introducen estrategias para el desarrollo del pensamiento histórico del alumnado? ¿Cómo se narra la historia?

La elección de estos parámetros se realizó en función del modelo conceptual del pensamiento histórico, pues interesó relacionarlo con las dinámicas de aula en tres aspectos: los contenidos, las actividades de enseñanza y las narrativas de la historia que se hacen en el aula. El diario de campo se implementó para describir todo aquello que sucedió durante la clase pero que no tenía relación ni con la enseñanza ni con el aprendizaje, aunque pudiera llegar a condicionarlos.

Para analizar los datos se utilizó la codificación temática y la codificación teórica (Flick, 2007), este procedimiento interesó porque la información se segrega en unidades de análisis que luego se agrupan en categorías y subcategorías, dando como resultado nuevos códigos que permiten explicar el objeto de estudio planteado.

La codificación se logró a través de tres etapas que dialogaron entre sí. La primera es la codificación abierta donde "se trata de expresar los datos y los fenómenos en forma de conceptos (...) se clasifican las expresiones por sus unidades de significado (palabras individuales, secuencias breves de palabras) para asignarles anotaciones y sobre todo «conceptos» (códigos)" (Flick, 2007, p. 193).

Posteriormente se procedió con la codificación axial, entendida como el proceso "de relacionar las categorías a subcategorías" (Strauss \& Corbin, 2002, p. 134). En esta fase se determinaron las propiedades y dimensiones de cada categoría para reagrupar los datos en torno a las subcategorías. Por último, se realizó la codificación selectiva, en la cual se determina la categoría central de todos los datos. Las etapas de la codificación no fueron secuenciales, al contrario, se construyeron en diálogo permanente a través del Método Comparativo Constante (MCC), el cual se refirió a las comparaciones que se hace de los datos hasta llegar a la saturación de la información (Verd \& Lozares, 2016).

Además, se realizó una triangulación de fuentes (docentes, estudiantes y currículo) para analizar las relaciones entre los datos obtenidos, esta estrategia nos permitió efectuar análisis comparativos y contraponer los discursos sobre los procesos de formación inicial docente.

Por último, el estudio de la formación inicial docente y el pensamiento histórico a través de las codificaciones teóricas y temáticas permitió relacionar la teoría con los datos obtenidos, de forma que se abren vetas para estudiar el desarrollo de los procesos de aprendizaje del futuro profesorado a través de metodologías centradas en la investigación cualitativa, para profundizar en contextos concretos.

Los resultados de la investigación se presentan en tres apartados: a) Las finalidades de las prácticas docentes universitarias en la enseñanza de la historia; b) Las prácticas docentes universitarias: enfoques y características, y c) El sistema está hecho para que fallemos: experiencias estudiantiles en su formación inicial. Las evidencias señalan que los propósitos docentes no están acordes con las metodologías implementadas en las lecciones de historia, lo cual tiene un impacto en el desarrollo del pensamiento histórico del futuro profesorado. 


\section{ANÁLISIS Y DISCUSIÓN DE LOS DATOS}

\section{Las finalidades de las prácticas docentes universitarias en la enseñanza de la historia}

Con los documentos curriculares se comparó la información a partir de las finalidades, los objetivos, las estrategias metodológicas y la evaluación de las materias de historia. En cuanto a las finalidades la evidencia muestra un énfasis en los cursos por enseñar las relaciones entre el pasado y el presente, el desarrollo de las habilidades propias de la historia como, por ejemplo, la multicausalidad y los análisis comparativos entre regiones y países. Hay una preocupación de las docentes por trascender la narración lineal de la historia y por centrarse en los procesos históricos que marcaron el pasado de las sociedades.

En los objetivos de los cursos los verbos que se utilizaron con más frecuencia fueron analizar, comprender, explicar, estudiar y evaluar y todos ellos se centraban en los distintos contenidos históricos de los cursos, por tanto, nos preguntamos: ¿Qué se enseña de estos contenidos? Para contestar esta pregunta se cuantificó la frecuencia de los temas propuestos en los cuatro cursos de historia que se analizaron, como se indica en la Tabla 1:

Tabla 1. Frecuencia de la presencia del contenido en los programas de los cursos de historia.

\begin{tabular}{|c|c|}
\hline Tema & Frecuencia de la mención (f) \\
\hline $\begin{array}{l}\text { Desarrollo social, cultural y económico de las } \\
\text { sociedades en el pasado }\end{array}$ & 6 \\
\hline $\begin{array}{l}\text { Transformaciones de la sociedad (rupturas en el } \\
\text { tiempo histórico) }\end{array}$ & 6 \\
\hline $\begin{array}{l}\text { Construcciones del pasado (relación de las Instituciones } \\
\text { con el Estado en la construcción de las naciones o } \\
\text { civilizaciones) }\end{array}$ & 5 \\
\hline $\begin{array}{l}\text { Procesos históricos (construcción del Estado nación, } \\
\text { resistencias e identidades) }\end{array}$ & 3 \\
\hline
\end{tabular}

Fuente: Elaboración propia.

En la tabla anterior se muestra que las temáticas de los cursos de historia se basan en el análisis de los cambios y las continuidades de las sociedades del pasado, y para ello se utiliza el razonamiento de la historia para presentar unos contenidos que trascienden el relato positivista.

Cabe señalar que tanto las finalidades como los objetivos son aspiraciones que se manifiestan también en las entrevistas que se realizaron a las docentes de historia. La intención que se persigue en los cursos de historia es crear aprendizajes críticos sobre el pasado basados en unos contenidos que trasciendan los datos factuales.

Las estrategias metodológicas que se exponen en los programas de los cursos, y que se desprenden del plan de estudios, pretenden que el estudiantado aprenda la materia a través de ejes temáticos, de la relación sincrónica y diacrónica entre las distintas sociedades, la historia-problema, el diálogo crítico entre docentes-estudiantes, la explicación magistral a partir de un problema central y la discusión de lecturas previamente asignadas. Todo lo 
anterior, se realiza a partir de preguntas-problemas que construye el estudiantado, trabajos grupales, la participación del alumnado en las discusiones de clase y exposiciones de lecturas o de temas asignados previamente.

En cuanto a la evaluación se espera que el estudiantado se prepare con las lecturas asignadas, demuestre habilidades de investigación, comunicación oral y escrita y que construya argumentos propios sobre los temas trabajados. Se aspira a que el futuro profesorado se aleje de explicaciones descriptivas, lineales y cronológicas para que piense históricamente y lo replique en las aulas colegiales. Se evalúa a partir de distintos rubros que varían poco entre los cursos analizados, por lo cual elaboramos un promedio con los porcentajes que se les asigna a cada uno de esos rubros (Fig. 1).

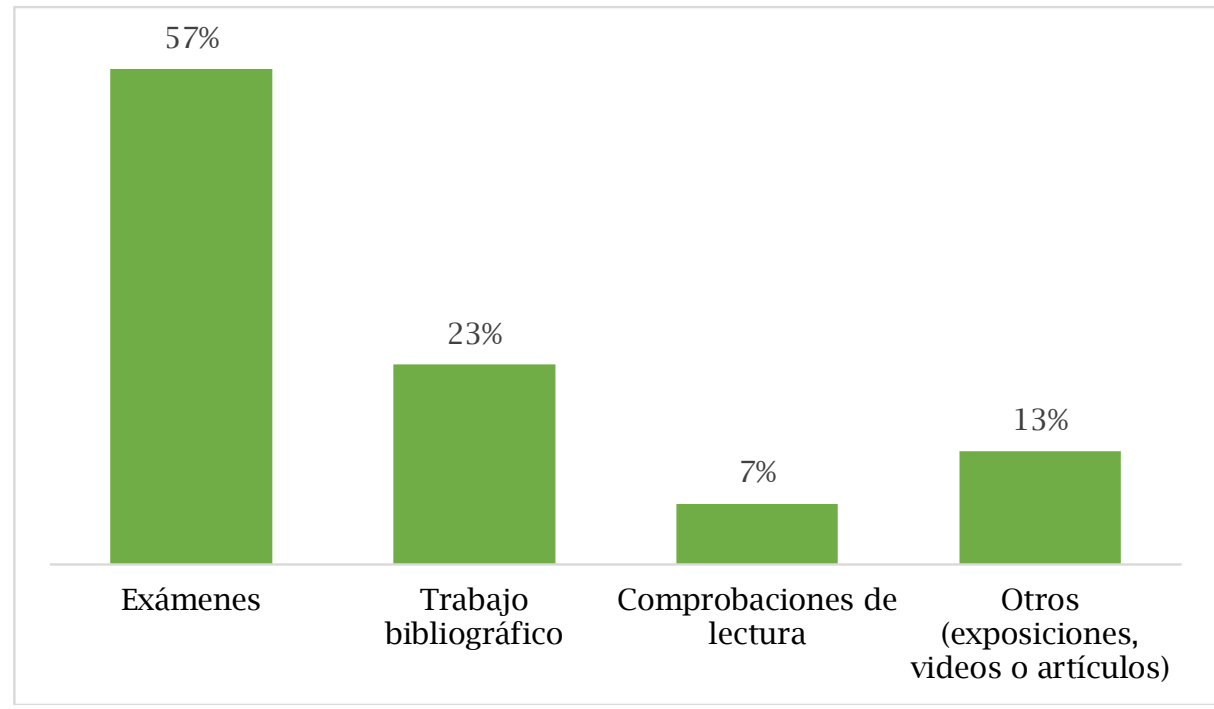

Fig. 1. Promedio de los porcentajes asignados a los rubros de evaluación en los cursos de historia de la carrera de Estudios Sociales.

Fuente: Elaboración propia a partir de los programas de curso de historia, 2019.

El concepto de evaluación que se deriva del gráfico anterior es el de demostración. El profesorado en formación debe demostrar el conocimiento adquirido a través de exámenes con preguntas abiertas sobre los procesos históricos vistos en clase. También debe desarrollar un trabajo bibliográfico en el que analice temáticas que la docente considera pertinentes de profundizar, por ejemplo, cuestiones culturales o de arte, religiosas, el papel de las mujeres, entre otros.

Las y los estudiantes deben demostrar que hicieron las lecturas asignadas a través de las comprobaciones de lectura. Los documentos que deben leer para cada curso tienen un promedio de entre 60 y 195 páginas por semana. Uno de los rubros con menor porcentaje asignado en las evaluaciones es donde el alumno o la alumna tiene mayor protagonismo: exposiciones, elaboración de videos o artículos, trabajos en clase que pueden ser grupales o individuales y construcción de preguntas-problema. El modelo evaluativo es sumativo y busca legitimar los aprendizajes a través de la cuantificación, por lo cual hay una discordancia entre las finalidades que se pretenden enseñar con la historia y la forma en la que se evalúa. 
Sobre lo anterior, González y colaboradores (2011) han mostrado que la evaluación del pensamiento histórico puede trascender la cuantificación y centrarse en el desarrollo de la criticidad sobre el pasado si éste se estudia desde las fuentes de información y la interpretación histórica. Además, los autores han entendido el proceso de enseñanza de la historia desde lenguajes narrativos y audiovisuales que le permiten al estudiantado analizar los contextos y las formas en las que imaginamos los acontecimientos sobre el pasado. En otras palabras, los métodos tradicionales de enseñanza y evaluación pueden superarse cuando el o la docente centra sus prácticas en estrategias que involucren al estudiantado en la construcción de la historia y que le permitan desarrollar sus propias narrativas sobre el pasado, por lo que la historia ha de permitirle al alumnado superar las ideas concebidas de la cultura para cuestionar los mitos y estereotipos de la vida en sociedad (Duquette, 2014), por lo cual, esa enseñanza siempre ha de estar en función de la justicia social y la construcción constante de la democracia.

\section{Las prácticas docentes universitarias: enfoques y características}

El pensamiento histórico es la aspiración por alcanzar de las docentes universitarias con sus estudiantes. Por lo anterior, con los datos obtenidos se caracterizaron sus prácticas docentes para profundizar la relación entre los propósitos que persiguen y cómo implementan los procesos de enseñanza. En la Tabla 2 se muestran los resultados:

Tabla 2. Características de las prácticas docentes universitarias.

\begin{tabular}{|c|c|c|c|c|}
\hline $\begin{array}{l}\text { Componentes } \\
\text { de la práctica } \\
\text { docente: }\end{array}$ & Docente 1 & Docente 2 & Docente 3 & Docente 4 \\
\hline $\begin{array}{l}\text { Los propósitos } \\
\text { que se } \\
\text { persiguen } \\
\text { son... }\end{array}$ & $\begin{array}{l}\text { analizar e } \\
\text { interpretar } \\
\text { la realidad } \\
\text { sociopolítica actual }\end{array}$ & $\begin{array}{l}\text { comprender la teoría de } \\
\text { la historia para aplicarla } \\
\text { en el análisis del pasado }\end{array}$ & $\begin{array}{l}\text { problematizar los } \\
\text { procesos históricos }\end{array}$ & $\begin{array}{l}\text { demostrar el dominio } \\
\text { de la materia y su } \\
\text { dimensión creativa en la } \\
\text { construcción de nuevo } \\
\text { conocimiento }\end{array}$ \\
\hline $\begin{array}{l}\text { El contenido } \\
\text { se trabaja... }\end{array}$ & $\begin{array}{l}\text { a partir de } \\
\text { problemas sobre el } \\
\text { pasado }\end{array}$ & $\begin{array}{l}\text { de forma lineal y } \\
\text { cronológica }\end{array}$ & $\begin{array}{l}\text { vinculando procesos } \\
\text { históricos }\end{array}$ & $\begin{array}{l}\text { problematizando las } \\
\text { lecturas asignadas cada } \\
\text { semana }\end{array}$ \\
\hline $\begin{array}{l}\text { La } \\
\text { metodología... }\end{array}$ & $\begin{array}{l}\text { se basa en la } \\
\text { clase magistral } \\
\text { con preguntas al } \\
\text { estudiantado }\end{array}$ & $\begin{array}{l}\text { combina trabajos } \\
\text { grupales con } \\
\text { exposiciones docentes }\end{array}$ & es expositiva & $\begin{array}{l}\text { combina exposiciones } \\
\text { del estudiantado, } \\
\text { preguntas sobre las } \\
\text { lecturas e intervención } \\
\text { de la docente }\end{array}$ \\
\hline $\begin{array}{l}\text { La evaluación } \\
\text { es... }\end{array}$ & $\begin{array}{l}\text { sumativa, centrada } \\
\text { en exámenes y } \\
\text { pruebas cortas }\end{array}$ & $\begin{array}{l}\text { sumativa, basada en } \\
\text { exámenes y pruebas } \\
\text { cortas }\end{array}$ & $\begin{array}{l}\text { sumativa y } \\
\text { formativa. Se busca } \\
\text { que el estudiante } \\
\text { aprenda a través } \\
\text { de los esquemas } \\
\text { de investigación } \\
\text { histórica. }\end{array}$ & $\begin{array}{l}\text { sumativa, su base son } \\
\text { los exámenes y las } \\
\text { pruebas cortas para } \\
\text { medir el nivel de lectura } \\
\text { del estudiantado }\end{array}$ \\
\hline
\end{tabular}

Fuente: Elaboración propia 
Según la tabla anterior, los propósitos del profesorado son variados y pretenden alcanzar un desarrollo cognitivo centrado en la problematización del presente a partir de la teoría, los procesos históricos y la realidad sociopolítica actual o la cultura. Las docentes 1 y 3 tienen unas intenciones muy cercanas al reconstructivismo social, donde se espera que el estudiantado sea capaz de comprender su presente en función del bienestar común. En este enfoque la interpretación individual del pasado, el presente y el futuro es la fuente del conocimiento (Schiro, 2013). Mientras que las docentes 2 y 4 tienen una intencionalidad más académica, donde se busca la comprensión de la sociedad a partir de las disciplinas.

Las prácticas docentes de las profesoras de historia son dinámicas y complejas, y transitan en varios enfoques. Y es en esa dinamicidad donde se observa el conflicto y las contradicciones entre el discurso y la práctica. Al comparar el tipo de contenido, las docentes 1,3 y 4 lo estudian desde el enfoque del reconstructivismo social: la intención es que el estudiantado interprete su entorno y, con ello, pueda intervenir en la sociedad. La docente 2 es consecuente con la intencionalidad de su práctica docente (academicista) y ubica el contenido de forma lineal y cronológica.

Según los datos, las contradicciones son más evidentes cuando se analizan los enfoques que explican cómo se da clase y cómo se evalúa. Las metodologías de las docentes 1 y 3 se imparten desde un enfoque académico, caracterizado por una enunciación instructiva del conocimiento, el cual deriva de la disciplina histórica. Esto a pesar de que los propósitos que se persiguen son la construcción del conocimiento por parte de sus alumnos y alumnas.

Según los datos, las docentes 2 y 4 se preocupan por establecer metodologías participativas, muy cercanas al enfoque de aprendizaje centrado en el estudiantado, aunque la intervención estudiantil es poca porque en las discusiones prevalece la opinión de las docentes, quienes son las protagonistas de la clase. Para todos los casos, la evaluación es sumativa y va de la mano con el enfoque academicista, el cual pretende objetivar los procesos de aprendizaje a través de la medición (Schiro, 2013).

En este sentido, las finalidades en los procesos de enseñanza no están acordes con las metodologías de clase ni la evaluación aplicada. En todos los casos, la clase expositiva prevalece en las prácticas docentes y la participación del estudiantado es minoritaria. La evaluación se enfoca en medir los resultados de las pruebas y trabajos asignados. En este sistema evaluativo si el estudiante se equivoca puede perder el curso, pues no existe la posibilidad de corregir los desaciertos. El aprendizaje no es visto como un proceso gradual de obtención del conocimiento.

Con respecto a la formación de docentes de Estudios Sociales, Adler (2008) afirma que los procesos de enseñanza deben situarse en la práctica de aula, para superar la brecha entre la Universidad y la Escuela, de esta forma, el centro de la FID no ha de ser la disciplina, sino cómo enseñarla. Con esta idea no negamos la trascendencia de los contenidos disciplinares, pero éstos constituyen sólo un medio y no el fin de los aprendizajes. Las contradicciones entre los propósitos y las prácticas docentes universitarias develan la necesidad de trabajar la competencia didáctica en la carrera de Estudios Sociales, para promover un currículo y unas lecciones de historia centradas en enseñar a enseñar. 


\section{El sistema está hecho para que fallemos: experiencias estudiantiles en su formación inicial}

Para ampliar la mirada sobre el desarrollo del pensamiento histórico en la carrera de Estudios Sociales, le preguntamos a los alumnos y alumnas en formación docente, cuáles son los desafíos de aprender historia (Fig. 2).

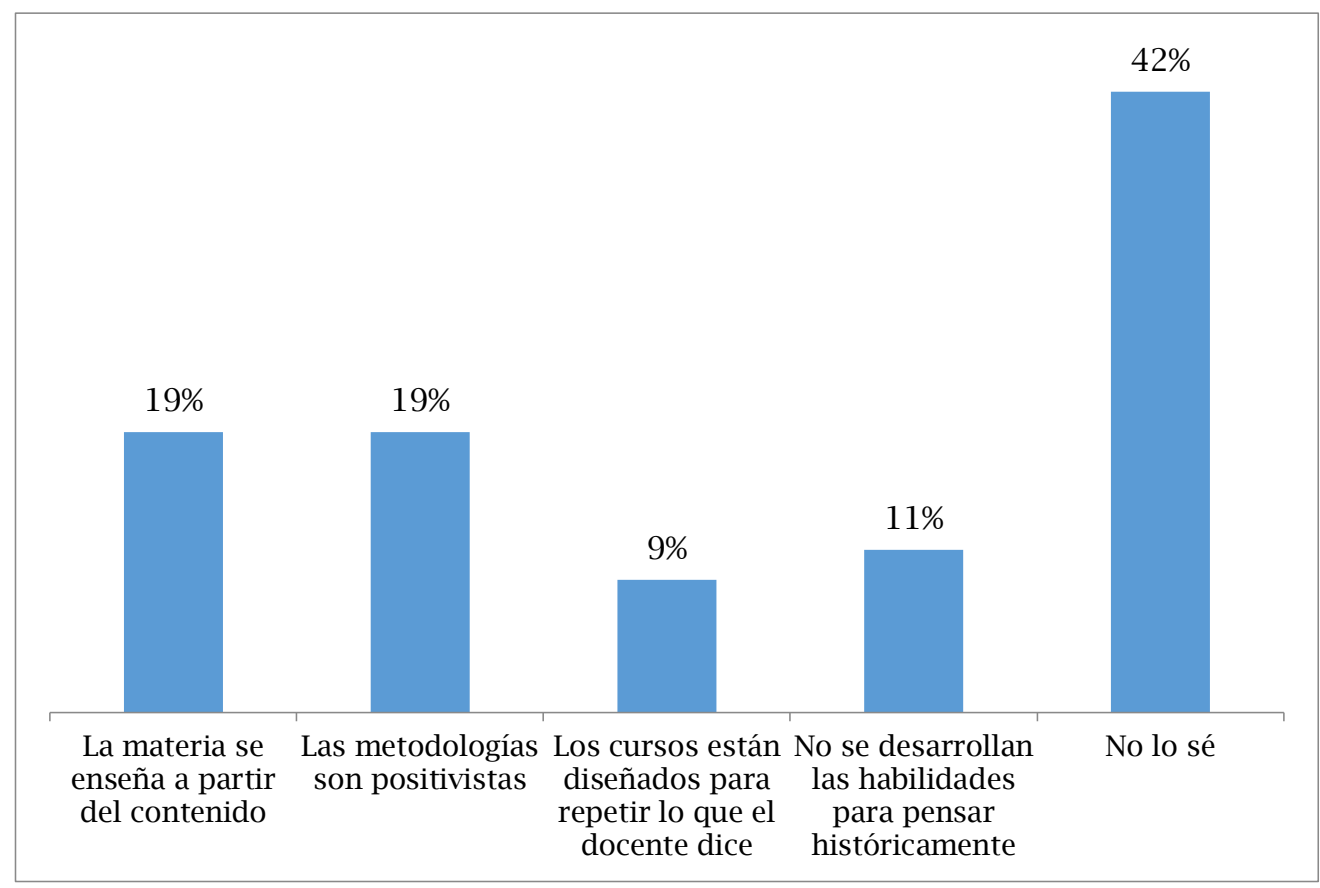

Fig. 2. Desafíos que enfrenta el estudiantado para aprender historia en la carrera de Estudios Sociales. Fuente: Elaboración propia a partir del cuestionario a II, III y IV nivel, 2019.

El 42\% no sabe cuáles son los problemas para aprender historia en la carrera de Estudios Sociales, un 19\% afirma que el mayor reto es que la materia se enseña a partir del contenido, otro 19\% dice que son las metodologías positivistas, el 9\% refiere a la repetición de los discursos docentes y un 11\% manifestó que no se desarrolla el pensamiento histórico. Cuando desagregamos los datos por niveles, la tendencia en las respuestas anteriores se mantuvo.

Tocante a lo anterior, y según las evidencias, las profesoras dan por un hecho que los estudiantes dominan los conceptos del pensamiento histórico. Palabras como sincrónico y asincrónico, anacronismos, continuidad, cambio, perspectiva histórica, significado histórico, periodización, entre otras, no se hacen explícitas en las lecciones de historia. El estudiantado debe resolver por sí mismo esos aprendizajes, porque, además, necesita demostrar su dominio en las evaluaciones. Un estudiante relató este desafío de la siguiente manera:

Yo siento que [los aprendizajes sobre el pensamiento histórico] lo dan como un hecho, como que usted no va a cometer ese tipo de errores. Varios profesores han regañado a varios compañeros o a mí me han regañado en Historia Universal al 
principio, por usar conceptos contemporáneos para [explicar] algo antiguo, entonces creo que lo dan como un hecho, como que usted ya trae esa habilidad desde que viene del colegio, se menciona como no vuelva a usar anacronismos, y le dicen qué era un anacronismo, pero hasta ahí y se da como un hecho y listo, entonces no se trabaja (Tobías, comunicación personal, 11 de junio de 2019).

En cuanto a lo anterior, aprender el pasado no es un acto natural (Wineburg, 2001), no es algo innato del ser humano, por ello, el o la docente en formación requiere procesos de enseñanza que impliquen como elementos básicos: a) las estructuras que conforman el lenguaje histórico y sus narrativas, b) las formas en las que se construye el conocimiento sobre el pasado y c) la interpretación histórica a través de las evidencias. En otras palabras, para aprender una historia crítica es necesario hacer historia, no sólo estudiar los resultados de investigaciones que, en materia escolar, terminan siendo contenidos estáticos del currículo.

Con respecto a esta idea, Santisteban y Pagès (2016) afirman que el estudio del pasado debe basarse en la interpretación de las evidencias, pues el dato factual por sí solo no resuelve los problemas sociales de la actualidad. El alumnado que viene del colegio tiene una noción de la historia, pero ésta debe ser complementada con la comprensión del pasado que trascienda el contenido de la materia.

Sobre la experiencia estudiantil en su formación inicial, y según las evidencias, podemos ubicar tres características en el discurso de las y los estudiantes: a) la institucionalización del estudiantado, b) la incertidumbre de fallar y c) la apropiación del lenguaje del historiador o historiadora. Con respecto a la primera característica, una estudiante definió como "institucionalización del estudiantado" la posición poco crítica de una parte del alumnado ante los procesos de enseñanza que recibe. De acuerdo con los datos, existe una resistencia para aprender de manera diferente. La posición de las y los estudiantes es pasiva en las clases de Historia, pues se limitan a tomar anotaciones en los cuadernos, participan cuando el o la docente abre el espacio y no cuestionan los contenidos vistos en clase.

En otras palabras, la institucionalización deriva de la idea de que algunos y algunas estudiantes están en una "zona de confort" con las actividades rutinarias de los cursos de historia: hacer lecturas semanales, comentarlas en clase, hacer las pruebas y presentar los trabajos.

Otra idea que manifestaron las y los estudiantes es la incertidumbre sobre su rol como docentes, debido a lo que consideran poca autonomía para dar clases de forma diferente. Tobías de último año de la carrera afirmó lo siguiente:

Muchas veces a uno se le enseña que el sistema [educativo] es perfecto y cuando uno va a dar lecciones se da cuenta de que el sistema está hecho para que fallemos, usted todo lo que quiera dar o como lo quiera dar no le va a funcionar porque el sistema está diseñado para eso, entonces los estudiantes [de colegio] no aprenden como uno quisiera que lo hagan (Tobías, comunicación personal, 11 de junio de 2019). 
Sobre esta idea, las críticas que se hacen entre las y los estudiantes es que no todas ni todos reconocen que son parte de una "institucionalización" que funciona como barrera para que sean conscientes de las estructuras laborales a las que se enfrentarán una vez que empiecen a trabajar en las aulas de secundaria o de los procesos de aprendizaje que reciben en la Universidad.

La última característica de la experiencia estudiantil es el uso de un lenguaje propio de los y las historiadoras para construir y validar sus argumentos. Por ejemplo, cuando el estudiantado se refería al estudio del pasado era recurrente que hablara de procesos históricos, del positivismo, de los anacronismos o que citara a autores como John Lynch, Leslie Bethell e incluso a Fukuyama. Con Gerardo, de I nivel, trabajamos un taller el día que entró a la Universidad, cinco meses después participó del grupo de discusión. La forma en la que él expresaba sus opiniones era diferente, pues trataba de explicarse a partir de autores, recurriendo a ideas de la Escuela de los Annales y la herencia de March Bloch, o de las consecuencias del positivismo en la investigación histórica (temas que había visto en el curso de Introducción a la Historia).

Por otra parte, sobre la percepción del profesorado universitario, las codificaciones realizadas indican dos tipos de docencia. La primera está centrada en la docente como protagonista y se caracteriza por:
a) lecciones aburridas donde no se integra al estudiantado,
b) practicar una evaluación coercitiva,
c) carecer de un ambiente de confianza para participar,
d) poca participación estudiantil por temor a ser ridiculizados.

El segundo tipo de docencia se determina por: a) las explicaciones de historia se entienden mejor, b) se utilizan diferentes estrategias de enseñanza, c) hay mayor participación estudiantil.

En este sentido, los obstáculos que enfrenta el profesorado en formación para el desarrollo del pensamiento histórico son: a) metodologías basadas en la transmisión del conocimiento o educación bancaria; b) evaluación sumativa; c) el profesor o profesora como centro del proceso de enseñanza; d) actitud pasiva del estudiantado; e) clases de pedagogía centradas en la psicología del aprendizaje donde no se estudian los diálogos entre la didáctica de las ciencias sociales y las disciplinas de referencia; f) conocimientos de historia, geografía y pedagogía fragmentados; g) prácticas docentes al final de la carrera, donde la experiencia de aula está disociada de los aprendizajes universitarios (brecha escuela-Universidad).

\section{CONCLUSIONES}

La evidencia señala que el profesorado universitario basa sus clases de Historia en métodos tradicionales, como la clase magistral y la evaluación sumativa. Estas metodologías aburren a las y los estudiantes de la carrera, quienes tienen una actitud pasiva en sus procesos de aprendizaje. Lo anterior, constituye uno de los mayores retos en las lecciones de Historia, 
pues se necesita trascender el contenido, para enseñar el pasado como una construcción que se hace desde el presente y, por ende, que responde al contexto y a los intereses en los que se desarrolla, como lo ha mostrado Duquette (2014) en sus investigaciones.

Los datos nos muestran que las y los docentes de historia asumen que el estudiantado sabe cómo aplicar los conceptos del método histórico a los análisis del pasado, esto lleva a que las lecciones de Historia se centren en el contenido y no en cómo se piensa o construye ese contenido. Como indica Pagès (2009), lo recomendable es partir de los significados del alumnado y de sus contextos, para propiciar aprendizajes que desarrollen el pensamiento histórico y, por ende, el pensamiento crítico. De esta forma, se propone construir mecanismos que permitan equilibrar el conocimiento experto con el conocimiento escolar, de forma que el alumnado de la carrera de Estudios Sociales aprenda a pensar por sí mismo la historia que enseñará.

Lo anterior, se relaciona con la forma en la que se analiza y jerarquiza el conocimiento disciplinar y el referente a su enseñanza. Ambos se diferencian por las finalidades que persiguen y las formas en las que se presentan los saberes (Pagès, 2018). Los datos no evidenciaron que estas diferencias se utilizaran en las lecciones de Historia para reflexionar el tipo de contenido que el estudiantado utilizará en sus clases de Estudios Sociales. En este sentido, es necesario diferenciar el conocimiento propio de la historia que se investiga, con el conocimiento pedagógico del contenido (Shulman, 2000) para contextualizar los aprendizajes del alumnado a su quehacer profesional.

Como sugiere Mattozzi (2015), el problema estriba en que el estudiantado aprende historia desde el conocimiento experto, pero el contenido que enseñará es escolar. Los datos señalan que el profesorado en formación no reconoce estas diferencias en sus prácticas de aula, por lo cual se continúa con la enseñanza de una historia tradicional.

La enseñanza del pensamiento histórico es un desafío en la carrera de Estudios Sociales, principalmente por las dificultades para comprender el pasado, las estrategias implementadas en las lecciones de Historia y la pasividad del estudiantado. Las contradicciones entre las finalidades y las prácticas docentes universitarias son uno de los retos de la carrera, aunque también una oportunidad para reflexionar y debatir sobre los propósitos de la formación inicial en la Escuela de Historia.

\section{REFERENCIAS}

Adler, S. (2008). The education of social studies teachers. En Levstik, L. (Ed.), Handbook of research in Social Studies (pp. 329-351). Reino Unido: Routledge.

Armento, B. (1996). The professional development of social studies educators. In J. Sikula et al. (Eds.), Handbook of Research on Teacher Education (pp. 485-502). Estados Unidos: McMillan.

Armento, B. (2000). El desarrollo profesional de los profesores de Ciencias Sociales. En J. Pagès, J. Estepa \& G. Travé (Eds.), Modelos, contenidos y experiencias en la formación del profesorado de Ciencias Sociales (pp. 19-39). España: Universidad de Huelva.

Chávez, C., \& Pagès, J. (2019). Habilidades de pensamiento histórico desarrolladas por 
estudiantes de formación inicial del profesorado de historia de Chile. En M. João, A. Dias \& N. De Alba (Coords.), Enseñar y aprender didáctica de las ciencias sociales: la formación del profesorado desde una perspectiva sociocrítica (pp. 776-788). Lisboa: Escola Superior De Educação.

Duquette, C. (2014). Through the Looking Glass: An Overview of the Theoretical Foundations of Quebec's History Curriculum. In R. Sandwell \& A. von Heyking (Eds.), Becoming a History Teacher. Sustaining Practices in Historical Thinking and Knowing (pp. 139-157). Canada: University of Toronto Press.

Flick, U. (2007). Introducción a la investigación cualitativa. España: Morata.

González, N., Santisteban, A., \& Pagès, J. (2011). ¿Cómo evaluar el pensamiento histórico del alumnado? En P. Miralles, S. Molina \& A. Santisteban (Eds.), La evaluación en el proceso de enseñanza y aprendizaje de las ciencias sociales (pp. 221-232). España: AUPDCS.

Libâneo, J. (2012). La integración entre conocimiento disciplinario y conocimiento pedagógico: una perspectiva para el trabajo profesional de los profesores. Revista Paulo Freire, $1(11), 21-38$.

Mattozzi, I. (1999). La transposición del texto historiográfico: un problema crucial de la didáctica de la historia. Teoría y Didáctica de las Ciencias Sociales, 4, 27-56.

Mattozzi, I. (2015). Una epistemología y una metodología de la historia para la didáctica. Diálogos, 19(1), 57-72.

Pagès, J. (2009). Enseñar y aprender ciencias sociales en el siglo XXI: reflexiones casi al final de una década. Investigación en Educación, Pedagogía y Formación Docente, II Congreso Internacional, 140-154. Colombia: Universidad Pedagógica Nacional, Universidad de Antioquia.

Pagès, J. (2018). Aprender a enseñar historia. Las relaciones entre la historia y la historia escolar. Trayectorias universitarias, 7(4), 53-59.

Plá, S. (2005). Aprender a pensar históricamente. La escritura de la historia en el bachillerato. México: PYV editores.

Ramírez, J., \& Pagès, J. (2019). Pensar históricamente en la Universidad: ¿Cómo aprende historia el futuro profesorado de Estudios Sociales? En M. João, A. Dias \& N. De Alba (Coords.), Enseñar y aprender didáctica de las ciencias sociales: la formación del profesorado desde una perspectiva sociocrítica (pp. 789-798). Lisboa: Escola Superior De Educação.

Santisteban, A., \& Pagès, J. (2016). La historia y la enseñanza de la historia. Un punto de vista desde la didáctica de la historia y de las ciencias sociales. En M. Jara \& G. Funes (Coords.), Didáctica de las ciencias sociales en la formación del profesorado. Perspectivas y enfoques actuales (pp. 63-86). Argentina: Universidad Nacional del Comahué.

Schiro, M. (2013). Curriculum Theory: Conflicting Visions and Enduring Concerns. Nueva York: SAGE.

Seixas, P., \& Morton, T. (2013). The Big Six Historical Thinking Concepts. Canadá: Nelson Education.

Shulman, L. (2000). Teacher Development: Roles of Domain Expertise and Pedagogical Knowledge. Journal of Applied Developmental Psychology, 1(21), 129-135.

Strauss, A., \& Corbin, J. (2002). Bases de la investigación cualitativa. Técnicas y procedimientos 
para desarrollar la teoría fundamentada. Colombia: Editorial Universidad de Antioquia. Verd, J., \& Lozares, C. (2016). Introducción a la investigación cualitativa. Fases, métodos y técnicas. Madrid: Editorial Síntesis.

Wineburg, S. (2001). Historical Thinking and other Unnatural Acts. Charting the future of teaching the past. Filadelfia: Temple University Press. 